\title{
Interrelation between inflammation, thrombosis, and neuroprotection in cerebral ischemia
}

\author{
Wendy J. van der Spuy ${ }^{1}$ and Etheresia Pretorius ${ }^{2, *}$ \\ ${ }^{1}$ Department of Anatomy, School of Medicine, Faculty of \\ Health Sciences, University of Pretoria, South Africa \\ ${ }^{2}$ Department of Physiology, School of Medicine, Faculty \\ of Health Sciences, University of Pretoria, Private Bag \\ x323, Arcadia 0007, South Africa \\ * Corresponding author \\ e-mail: resia.pretorius@up.ac.za
}

\begin{abstract}
Stroke by mechanism of thrombotic cerebral ischemia is one of the leading causes of death and/or disability worldwide. Current research is under consensus that there are sex-based differences in both the prevalence and presentation of stroke and thrombosis. Here we discuss the interrelation between thrombosis and inflammation and call attention to points in the cerebral ischemic cascade where estrogen may be involved in neuroprotection. Cerebral ischemia triggers a series of events including inflammation, which is deeply interrelated with thrombosis; inflammation not only produces local thrombosis, but thrombosis can also amplify inflammation especially through the synergism of leukocyte and platelet activity. Research involving experimental animal models of cerebral ischemia has shown that sex hormones, especially estrogen, offer a degree of neuroprotection. Mechanisms of this neuroprotection may be linked to certain anti-inflammatory properties of estrogen, as well as estrogen's regulation of thrombosis through the lowering of coagulation factors, among others. It is also understood that sex hormones alter the function and morphology of platelets and fibrin networks, and changes in platelet and fibrin network morphology offer one of the earliest confirmations of inflammation. Sex hormone levels, inflammatory processes, and thrombotic mechanisms are profoundly interconnected in predicting the outcome of cerebral ischemia.
\end{abstract}

Keywords: anti-inflammatory; coagulation; estrogen; leukocytes; platelets.

\section{Introduction}

Stroke is currently the second leading cause of death and/or disability worldwide, and the existence of a larger aging population suggests that stroke research will become more important in the future (Elkins and Johnston, 2003; Braeuninger and Kleinschnitz, 2009). Thrombotic cerebral ischemia accounts for more than half of all cases of stroke worldwide. It results from an occlusion of cerebral vasculature, reducing or preventing the supply of oxygen to the cerebrum, thus hampering metabolic demand, and ultimately leading to death of brain tissue by ischemic stroke.

Research suggests that there are sex-based differences in the prevalence and presentation of both stroke and thrombosis (Bailey et al., 2009). Sex hormones not only alter procoagulant protein expression (Lowe et al., 2004) and the function of blood and vascular cells (Kadir et al., 1999; Butenas and Mann, 2002; Leng et al., 2004), but differences in platelet function (Liao et al., 2001; Suzuki et al., 2009) and in thrombosis activity (Bailey et al., 2009) have also been noted. Estrogen is to a degree neuroprotective (Liao et al., 2001; Suzuki et al., 2009; Selvamani and Sohrabji, 2010) in certain cases of induced cerebral ischemia, as females appear to suffer less severe consequences of stroke, including lesser neural tissue loss, than their male counterparts (McCullough and Hurn, 2003; Suzuki et al., 2009). In the absence of ovarian hormone production at menopause, females are again at higher risk to strokes than their male counterparts, and this risk continues to increase with age, as women have a longer life expectancy than men (Mitka, 2006; Suzuki et al., 2009). Thus, both sex and age play an important role in the occurrence of thrombotic events and the severity of neural damage subsequent to a stroke.

Platelets and fibrin play an important role in the normal coagulation process where they are involved in the maintenance of hemostasis (Herd and Page, 1994). Their activation may be due to damage of the vessel wall or activation of the endothelium by chemicals, cytokines, and also inflammatory processes (Camera et al., 1999; Butenas and Mann, 2002). Activated platelets synthesize/secrete inducers of platelet aggregation and adhere to the injured vessel wall, as well as aggregate to each other in order to form a platelet-rich plug or thrombus, which secures hemostasis; this plug is then stabilized by fibrin formation as fibrinogen is activated by binding to activated platelets. Fibrinogen is the major plasma protein coagulation factor (Lowe et al., 2004), and though plasma levels thereof are decreased by estrogen during the menstrual cycle, these levels are known to be higher in females than in males (Bailey et al., 2009). Although fibrin forms the core matrix of a thrombus, its structure depends also on the cellular elements embedded in its meshwork and the overall rate of coagulation reactions initiated by platelet aggregation (Wohner, 2008). Morphological changes of fibrin networks may therefore occur due to several kinetic and modulating factors present in plasma.

A thrombotic event is associated with a change in hemostasis and cellular components that play a fundamental role 
in blood platelets and fibrin network formation. It is well known that thrombotic events are the most common cause of stroke and resultant cerebral ischemia (Braeuninger and Kleinschnitz, 2009). Furthermore, it is known that cerebral ischemia triggers a cascade of inflammatory processes, among others (Herd and Page, 1994; Gibson et al., 2005; Wang et al., 2007). Inflammation again causes an alteration in platelet activation (Camera et al., 1999; Butenas and Mann, 2002) and possibly further thrombotic events. It is suggested that estrogen provides neuroprotection through certain antiinflammatory mechanisms, among others (Vegeto et al., 2008; Suzuki et al., 2009).

\section{Cerebral ischemia}

With two-thirds of deaths from stroke complications occurring in developing regions of the world, such as sub-Saharan Africa, stroke is the second leading cause of death worldwide. The existence of a larger aging population suggests that stroke research will become more important in the future (Elkins and Johnston, 2003; Wang et al., 2007; Braeuninger and Kleinschnitz, 2009).

Stroke incidence and resultant cerebral ischemia can be linked to coagulation processes. The cascade of blood coagulation is initiated when subendothelial tissue factor is exposed to the flow of blood subsequent to damage or activation of the vessel endothelium by chemicals, cytokines, or inflammatory processes (Butenas and Mann, 2002). The formation of a thrombus at a site of vessel injury is thus a hemostatic process. A thrombotic event, however, is associated with a change in hemostasis and cellular components that play a fundamental role in blood platelet and fibrin network formation. Overactivity of any one component of the coagulation cascade can result in the formation of tight and rigid fibrin networks (Fatah et al., 1992), which can cause blockage of one or multiple cerebral blood vessels, resulting in cerebral ischemia.

Cerebral ischemia is known to trigger a series of complex events initiating with cerebral hypoperfusion and comprising bioenergetic failure of cellular components, excitotoxicity, oxidative stress, biphasic dysfunction of the blood-brain barrier, microvascular injury, hemostatic activation, inflammation, and formation of edema, as well as apoptosis and ultimate necrosis of neuronal, glial, and endothelial cells. This cascade of events is dependent on variables, such as onset and duration of ischemia, effectiveness of reperfusion, and resultant infarct size or tissue loss (Danton and Dietrich, 2003; Gibson et al., 2005; Brouns and De Deyn, 2009; Saenger and Christenson, 2010).

Blood-brain barrier disruption in ischemic stroke appears to be dependent on the response to and aggressiveness of reperfusion. Increased permeability of the blood-brain barrier takes place within the first 24 hours of an ischemic event, with further damage occurring 48-72 hours after ischemia in the absence of sufficient reperfusion (Saenger and Christenson, 2010). Inflammation itself is recognized as a key element of the pathological progression of ischemic stroke. The destructive or beneficial nature of inflammation seems to be dependent on the severity of ischemia. It is thus likely that early inflammatory responses may potentiate ischemic injury, whereas late responses may be beneficial to recovery and repair of ischemic lesions (Wang et al., 2007). Inflammation and thrombosis are deeply interrelated, as not only can inflammation produce local thrombosis, but thrombosis can also amplify inflammation (Libby and Simon, 2001).

\section{Thrombosis}

Thrombosis, the most common cause of stroke, is influenced by factors including endothelial injury, blood stasis or turbulent flow, and hypercoagulability of blood (Myers and Wakefield, 2005). Endothelial injury is the most common cause of hemostatic coagulation processes, and it is vital that these processes are understood. Endothelial damage to vasculature initiates a local inflammatory response, promoting a state of prothrombosis, which is driven by tissue factor, adhesion molecules, and proinflammatory cytokines and prothrombotic microparticles (Libby and Simon, 2001). Various disease states are also found to promote tissue factor exposure within vascular walls to blood flow, leading to the initiation of non-hemostatic coagulation processes (Mackman, 2004; Myers and Wakefield, 2005). Thrombosis and inflammation are interrelated (Stewart et al., 1974; Myers and Wakefield, 2005), as inflammation produces local thrombosis, and thrombosis can amplify inflammation (Libby and Simon, 2001). A thrombus, whether formed through hemostatic or non-hemostatic mechanisms, consists of platelets and fibrin as well as trapped red and white blood cells, which stabilize its structure.

Platelets and fibrin play an important role in the coagulation process, where they are involved in the maintenance of hemostasis. Platelets have a life span of 8-12 days, and though devoid of a nucleus, possess many features of classical inflammatory cells. Like neutrophils, they can undergo chemotaxis (Zhang et al., 1993), phagocytose foreign particles, contain and release adhesive proteins, activate complement, interact with foreign bodies, alter vascular tone, enhance vascular permeability, as well as store and metabolize various vasoactive substances, and release inflammatory mediators (Herd and Page, 1994). Structurally, platelet surfaces consist of a typical bilayer membrane composed of lipids, proteins, and carbohydrates. Surface glycoproteins are essential to their function and play a primary role in their adhesion to exposed subendothelial matrix proteins, interaction with thrombin, and exposure of fibrinogen receptors to facilitate aggregation (Roth, 1992; Herd and Page, 1994). Internally, platelets are only capable of limited protein synthesis, and few mitochondria are present, which contribute to energy metabolism of the platelet. In addition, a random cytoplasmic distribution of lysosomes, glycogen granules, and peroxisomes are present (Herd and Page, 1994). Following activation, platelets change shape from a discoid to a spherical form. This process is mediated by a contractile microtubular system, morphologically characterized by an 
extension of dendritic pseudopodia (White, 1987; Herd and Page, 1994).

Platelets are activated by a number of stimuli resulting in the expression and/or activation of surface receptors, secretion of vasoactive substances, adhesion, aggregation, and finally, thrombus formation. The activation may be due to damage of the vascular wall or activation of the endothelium by chemicals, cytokines, and also inflammatory processes (Camera et al., 1999; Butenas and Mann, 2002). Upon activation, platelets cover the exposed subendothelial matrix and mediate additional platelet and leukocyte recruitment through the release of microparticles that mediate local leukocyte-leukocyte and leukocyte-endothelial cell interactions - mechanisms which play a role in both thrombosis and inflammation (Wagner and Burger, 2003). Platelets are essential in the initial stages of thrombus formation because they adhere and aggregate at sites of vascular wall injury and then serve as a surface for coagulation reactions; the overall rate of which determines the final structure of fibrin (Wohner, 2008). Thrombi thus form locally in a vessel when injury occurs or endothelial activation takes place - as a hemostatic mechanism to repair the insult. Platelets, during adhesion to endothelium, are activated and release proinflammatory cytokines that further stimulate the endothelium (Weber and Springer, 1997; Wagner and Burger, 2003), promote hemoattraction of leukocytes, stimulate smooth muscle cell and fibroblast proliferation, promote collagen synthesis, and thus contribute directly to lesion progression and maturation (Ross, 1985; Wagner and Burger, 2003).

Fibrinogen is the major plasma protein coagulation factor and the best known precursor of fibrin, playing an important role in platelet aggregation by linking activated platelets, and therefore playing a key role in hemostasis and thrombosis. Activated platelets synthesize/secrete inducers of platelet aggregation, adhere to the injured vessel wall, and aggregate to each other in order to form a platelet-rich plug that secures hemostasis; this plug is then stabilized by fibrin formation as fibrinogen is activated by binding to activated platelets. Thus, not all circulating fibrinogen is functional or clottable. As the tissue repair process takes place, the fibrin plug is digested by fibrinolytic enzymes (Lowe et al., 2004). On the one hand, low levels of plasma fibrinogen are associated with an increased risk for bleeding, as platelet aggregation as well as fibrin plug formation is impaired. Elevated fibrinogen levels, on the other hand, may well be associated with the risk of stroke (Danesh et al., 2005), as elevated fibrinogen synthesis is inclined to shift the hemostatic balance in favor of coagulation/thrombosis.

Interestingly, thrombi are found to form readily and rapidly in the complete absence of fibrinogen in animal models. However, these thrombi are unstable and fail to resist shear stress, resulting in frequent thromboembolization, with downstream vessel occlusion (Ni et al., 2000; Wagner and Burger, 2003). Fibrinogen/fibrin complexes are thus required to secure thrombus stability for anchorage to the site of injury. In addition, this stability is dependent on fibrinogen/fibrin interaction with platelet integrin (a surface protein), which also slows down the growth of the thrombus (Hawiger, 1995; $\mathrm{Ni}$ et al., 2000; Wagner and Burger, 2003). Fibronectin is known to support platelet adhesion and distribution (Hynes, 1990; Wagner and Burger, 2003). Deficiency of plasma fibronectin though does not affect initial platelet adhesion (Sakai et al., 2001; Wagner and Burger, 2003), but delays thrombus formation quite substantially as platelets are continuously shed. Therefore, fibronectin is an important mediator of plateletplatelet interactions within thrombi as they form and grow. This mediation takes place through fibronectin's rapid binding to activated integrins, thus cross-linking platelets. Fibrin is then generated, which anchors the growing thrombus to the site of vascular injury (Ni et al., 2000; Wagner and Burger, 2003).

Platelet adhesion and activation are thus regulated by specific proteins on the platelet surface, and fibrinogen as well as fibronectin plays a fundamental role in the coagulation process (Ni et al., 2000; Lowe et al., 2004). Fibrin assembly (through the coagulation pathway and involvement of the platelets) from fibrinogen proceeds in a highly ordered fashion. Fibrin forms a network, which functions to stabilize the primary platelet plug. Although fibrin forms the core matrix of a thrombus, its structure depends also on the cellular elements embedded in its meshwork. Morphological changes of fibrin networks may therefore occur due to several kinetic and modulating factors present in plasma. Fibrinolysis plays an important role in hemostasis too. Leukocyte-derived enzymes, such as elastase, influence fibrinolysis by direct digestion of fibrin or indirectly modulating it by partial degradation of zymogens and inhibitors of coagulation and fibrinolytic proteases (Wohner, 2008).

Thrombosis research has shown that sex hormones have complex effects on vascular walls, coagulation proteins, and platelets - all which may alter thrombosis. In line with this, females have shown cyclic patterns in their coagulation proteins, which correspond to menstrual cycle patterns (Kadir et al., 1999; Bailey et al., 2009); in addition, they possess slightly higher fibrinogen levels than their male counterparts, though estrogen is known to decrease fibrinogen plasma levels (Mendelsohn and Karas, 1999; Bailey et al., 2009). There also seem to be sex-related differences in platelet function. It is known that isolated female platelets bind more fibrinogen and have a greater maximal aggregation extent than male platelet isolates, and this platelet reactivity is altered in ovariectomized females (Leng et al., 2004; Bailey et al., 2009). Males have been shown to have higher platelet counts and faster clotting times than their female counterparts, thus making them more susceptible to thrombosis - possibly due to the differences in growth hormone secretion between the sexes (pulsatile in males, sustained in females), which in turn influence protein production of coagulation and thrombosis regulators (Wong et al., 2008; Bailey et al., 2009). Isolates of male rat platelets have been shown to display greater maximal aggregation in vitro than platelets isolated from female rats; this aggregation is reduced in male rats that have undergone castration (Emms and Lewis, 1985; Bailey et al., 2009). It has been noted that both megakaryocytes and platelets express the estrogen receptor beta (ER $\beta$ ) as well as the androgen receptor (Jayachandran and Miller, 2003; Bailey et al., 2009); thus, 
it is almost certain that the sex hormones have an effect on thrombosis. Estrogen is hypothesized to have a direct effect on platelet function, whereas androgen seems to regulate megakaryocyte biology and platelet production (Peters et al., 2002; Bailey et al., 2009).

The occurrence of thrombosis is thus hemostasis in the wrong place, which results from local activation of platelets and coagulation, and also from increased concentrations of plasma fibrinogen (Lowe et al., 2004). As mentioned, females have elevated fibrinogen levels compared to their male counterparts, though estrogen does lower fibrinogen levels (Mendelsohn and Karas, 1999; Bailey et al., 2009), and in menopause, these plasma-lowering effects of estrogen are ruled out, rendering fibrinogen levels even higher than in cyclic females (Lowe et al., 1997, 2004). Many diseases like cancer, thrombotic disease, bleeding disorders, asthma, and even conditions like HIV/AIDS are associated with changes in platelet and fibrin structure. Fibrin structure itself has been shown to play a role in the development of vascular complications (Pretorius et al. 2006, 2007).

Cerebral ischemia may be a consequence of thrombosis when a change in hemostasis occurs, for example, in the instance of a blood disorder or diminished blood flow (due to age or even normal thrombus formation), resulting in a local accumulation of coagulation factors and thus increasing platelet aggregation. These changes in hemostasis thus alter the coagulation cascade and result in the formation of rigid fibrin networks, which do not digest as programed. These rigid thrombi may cause ischemia locally if they diminish or inhibit the blood flow in a vessel for an extended period, or pieces thereof may break free, termed a thromboembolus, and become lodged in another vessel where they can cause ischemia by inhibition of blood flow.

\section{Inflammation}

Inflammation is characterized by interactions among endothelial cells, platelets, and leukocytes, and causes endothelial activation regardless of the mechanisms by which inflammation itself was activated. Endothelial activation sets off the cell adhesion cascade, which results in the adherence and aggregation of platelets, chemokine deposition by platelets on the activated endothelial surface, the expression of cell adhesion molecules by endothelial cells and platelets, and ultimately, activation of leukocytes. Chemokines activate leukocytes, and further binding to adhesion molecules mediate the process of leukocyte rolling (on the activated 'sticky' endothelium), adhesion (through binding to fibrinogen), and transmigration into the subendothelial tissue (Butcher, 1991; Springer, 1994; Diacovo et al., 1996; Kuijper et al., 1996; Weber and Springer, 1997; Konstantopoulos et al., 1998; Wagner and Burger, 2003). Platelets are thus central to both thrombosis and inflammation.

The objective of inflammation is therefore to recruit leukocytes rapidly to a site of vascular injury. Endothelial dysfunction or injury promotes activation of the coagulation cascade by exposure of tissue factor (Gimbrone, 1995; Day et al.,
2005; Myers and Wakefield, 2005), as well as the activation of inflammatory process (Laursen et al., 2001; Altman, 2003; Myers and Wakefield, 2005). Inflammatory mediators then promote coagulation through further elevation of tissue factor (Drake et al., 1989; Esmon, 2003), which elevates fibrinogen synthesis, and fibrinogen levels will continue to rise under inflammatory conditions (Taylor et al., 1987; Esmon, 2003) unless hemostatic factors counteract this. Tissue factor is a membrane-bound protein that functions as a procoagulant (Libby and Simon, 2001), triggering thrombin generation, which then prompts activation of the coagulation cascade (Nemerson, 1988; Mann et al., 1998; Myers and Wakefield, 2005). Thrombin in turn amplifies the inflammatory response by activating the endothelium, resulting in the formation of more tissue factor (Pendurthi et al., 1997; Miller et al., 1998; Esmon, 2003) and high levels of platelet-activating factor (Bar-Shavit et al., 1986; Esmon, 2003), which is a neutrophil agonist (Lorant et al., 1991; Esmon, 2003) enhancing leukocyte activation and adhesion, as well as increasing inflammatory cytokines (Henn et al., 1998; André et al., 2002, Esmon, 2003). Inflammatory cytokines have been shown to increase platelet reactivity, which increases thrombogenic potential (Burstein, 1997; Esmon, 2003), further linking inflammation and thrombosis.

Monocytes do not express tissue factor unless they are stimulated by inflammatory mediators to transcribe the gene for tissue factor (Wilcox et al., 1989; Brand et al., 1991; Libby and Simon, 2001); thus, their recruitment and activation can lead to thrombogenesis. Activated monocytes that express tissue factor on their surfaces (Rauch and Nemerson, 2000) facilitate monocyte-platelet and monocyte-endothelial interactions through binding mechanisms of cell adhesion molecules (Wakefield et al., 1997; Shebuski and Kilgore, 2002; Myers and Wakefield, 2005). These interactions, driven by inflammatory mediators and tissue factor, lead to accelerated fibrin formation and deposition into a developing thrombus (Shebuski and Kilgore, 2002; Myers and Wakefield, 2005). Thus, the specific interaction between cell adhesion molecules and their leukocyte receptors is what stimulates fibrin formation (Goel and Diamond, 2001; Myers and Wakefield, 2005), and procoagulant microparticles derived both from activated leukocytes and platelets amplify the coagulation process (Frenette et al., 2000; Myers and Wakefield, 2005).

Like endothelial cells and activated monocytes, smooth muscle of blood vessels can express tissue factor when exposed through endothelial breakage, thus also contributing to thrombogenesis (Schecter et al., 1997; Libby and Simon, 2001). Besides production of procoagulant tissue factor, the smooth muscle can also undergo inflammatory activation when exposed to thrombin and products of thrombosis (Kranzhöfer et al., 1996; Libby and Simon, 2001), thus amplifying the inflammatory response and promoting systemic procoagulant effects due to increased fibrinogen levels in circulation (Libby and Simon, 2001).

The progression of the inflammatory response subsequent to a stimulus hence reflects a balance between prothrombotic and anticoagulant activities. The ability of proinflammatory cytokines to downregulate antithrombotic proteins and 
upregulate prothrombotic proteins shifts this balance toward a procoagulant state (ten Cate et al., 1997; Myers and Wakefield, 2005); in addition, these cytokines induce the immune defense mechanism and mediate leukocyte recruitment. Inflammatory cells are important to the process of thrombus recanalization and organization. Although it may seem intuitive that a decrease in inflammation will decrease thrombogenesis, once a clot forms, the presence of neutrophils is important for recanalization (Varma et al., 2003; Myers and Wakefield, 2005). Accordingly, inflammation leads to an imbalance between the pro- and anticoagulant properties of endothelium that can lead to local stimulation of the coagulation cascade (Nathan, 2002; Wagner and Burger, 2003). Early inflammatory responses may consequently contribute to damage, whereas late/delayed inflammatory responses are necessary to facilitate repair.

As mentioned previously, cerebral ischemia triggers inflammatory processes (Herd and Page, 1994; Gibson et al., 2005; Wang et al., 2007). The first inflammatory cells that enter the brain subsequent to trauma are neutrophils, followed by monocytes, and later on, resident microglia, astrocytes, and neurons are also activated (Morganti-Kossmann et al., 2001). Leukocytes and, after a few hours post-injury, microglia secrete proinflammatory cytokines and chemokines, the severity of which play detrimental roles in the pathophysiology of stroke (Morganti-Kossmann et al., 2001; Wang et al., 2007; Suzuki et al., 2009). Significant leukocyte influx into cerebral parenchyma and tissue remodeling are characteristics of cerebral ischemia/reperfusion (Barone et al., 1995; Wang et al., 2007). Interestingly, infarct volume has been shown to be reduced significantly through the inhibition of neutrophil infiltration, as it is evident that neutrophils wield the most damage to ischemic lesions once reperfusion is undertaken (Connolly et al., 1996; Guha and Mackman, 2001; Wang et al., 2007).

In areas of ischemia/reperfusion injury, platelets colocalize with leukocytes - an interaction linking hemostatic thrombotic and inflammatory responses (Ostrovsky et al., 1998; Libby and Simon, 2001). The inflammatory reaction subsequent to cerebral ischemia is characterized by neutrophil adherence to blood vessels 4-6 hours after onset of ischemia and their infiltration into the neural tissue with subsequent release of proinflammatory mediators, potentiating injury (Hallenbeck, 1996; Wang et al., 2007), by resultant accumulation and activation of monocytes in the area of lesion. Platelets promote accumulation of both neutrophils and monocytes at sites of injury, and neutrophil-platelet aggregates specifically influence cellular responses by inducing further leukocyte activation, enhancing cell-adhesion molecule expression, and generating signals that promote platelet integrin (surface protein) activation and chemokine synthesis (Ott et al., 1996; Furman et al., 1998; Libby and Simon, 2001). Chemokines stimulate cytoskeletal reorganization of neutrophils and monocytes to facilitate their motility, proliferation of fibroblasts and astrocytes for glial scar formation, apoptosis and necrosis of neurons, and the phagocytic ability of macrophages and microglia to remove the debris of damaged tissue (Morganti-Kossmann et al., 2001).

\section{Neuroprotection}

Sex hormones target the central and peripheral nervous systems, affecting brain development and differentiation and influencing neuronal functions (Manthey and Behl, 2006; Drača, 2009). In humans, it is accepted that premenopausal or cyclic women present with a lower incidence of ischemic stroke than men; this distinction is, however, no longer present when postmenopausal or acyclic women are compared to men. Furthermore, the ischemic stroke risk increases in both sexes with age (Wolf, 1990; Gibson et al., 2005; Braeuninger and Kleinschnitz, 2009). Thus, sex hormones must have a role in neuroprotection and the decline thereof with age.

Experimental animal studies have not only reported that young females present with smaller cerebral infarcts and thus less neural tissue injury than their male counterparts (Alkayed et al., 1998; Braeuninger and Kleinschnitz, 2009), but also that high endogenous estradiol levels during the estrus cycle seem to correlate with smaller infarct size in females (Carswell et al., 2000; Braeuninger and Kleinschnitz, 2009). This advantage is abolished in ovariectomized animals, due to the loss of endogenous female sex hormones (Simpkins et al., 1997; Alkayed et al., 1998, 2000; Hawk et al., 1998; Liao et al., 2001; Gibson et al., 2005; Park et al., 2006; Drača, 2009; Selvamani and Sohrabji, 2010), and the consequences of cerebral ischemia in aged animals are more severe than in young animals (Davis et al., 1995; Alkayed et al., 1998).

The female sex hormone $17 \beta$-estradiol has been shown to be the principal circulating estrogen protecting the brain from damage, by reducing infarct size after experimental cerebral ischemia, through attenuation of markers of apoptosis by activation of mediators of cell survival signaling pathways. It seems that when administered several days before inducing cerebral ischemia, physiological levels of estradiol attenuate brain injury through the suppression of neuronal apoptosis and genomic actions by acting through mechanisms of the classical nuclear estrogen receptors (Liao et al., 2001; Prewitt and Wilson, 2007; Jia et al., 2009; Suzuki et al., 2009); this is, however, not the case with acute administration of $17 \beta$-estradiol at the time of injury, as this does not reduce the extent of infarction (Dubal et al., 1998; Suzuki et al., 2009).

In studies of the neuroprotective extent of estradiol, researchers have shown that a single high-dose injection $(1 \mathrm{mg} / \mathrm{kg}$ ) of $17 \beta$-estradiol administered to male rats immediately before experimental cerebral ischemia was capable of reducing cortical tissue loss, and that castration of male rats, resulting in the loss of testosterone, did not alter these results (Toung et al., 1998). Moreover, injection of exogenous $17 \beta$-estradiol was only neuroprotective in the male brain, revealing that endogenous estrogen is sufficient to protect the female brain and that exogenous $17 \beta$-estradiol had no additional protective effect (Toung et al., 1998). Thus, the hypothesis that estrogen is a major mediator of sex differences displayed in stroke is heavily strengthened (Drača, 2009).

$17 \beta$-Estradiol salvages the brain from ischemic injury, even enhancing recovery and reducing infarct size in ovariectomized (Simpkins et al., 1997; Selvamani and Sohrabji, 2010) and reproductively senescent or aged females (Alkayed et al., 
2000; Liao et al., 2001), as well as in male animals (Hawk et al., 1998). In the case of acyclic females, this is subject to administration of $17 \beta$-estradiol at the onset of senescence or ovariectomy and not in older acyclic females (Bake and Sohrabji, 2004; Suzuki et al., 2009; Selvamani and Sohrabji, 2010). This seems to be due to the upregulation of estrogen receptor alpha $(\mathrm{ER} \alpha)$ close to the onset of senescence, in response to declining estrogenic stimuli from the ovaries, inadvertently providing a substrate for exogenous estrogen. However, in older acyclic females, the ER $\alpha$ is already downregulated, and thus, exogenous estrogen becomes deleterious (Jezierski and Sohrabji, 2001; Selvamani and Sohrabji, 2010). Ischemic injury itself has been found to increase the expression of ER $\alpha$ in the cerebral cortex, without influencing ER $\beta$ expression. Consequently, it is believed that it is this $E R \alpha$ re-expression after ischemic injury that mediates $17 \beta$-estradiol's profound neuroprotection against ischemia (Dubal et al., 2001; Suzuki et al., 2007, 2009).

Cerebral ischemia triggers a complex series of events, including excitotoxicity, inflammation, and formation of edema, as well as apoptosis and necrosis (Danton and Dietrich, 2003; Gibson et al., 2005; Saenger and Christenson, 2010) - all of which are reduced by estradiol through free radical scavenger action, among others (Demopoulos et al., 1972; Singer et al., 1996; Gibson et al., 2005; Drača, 2009). Estradiol seems to target neural cells by indirect transcriptional mechanisms and by direct mechanisms, stabilizing neurotransmission, inhibiting apoptosis, reducing cerebral edema, and exerting anti-inflammatory and antioxidant effects (Manthey and Behl, 2006; Drača, 2009). Figure 1 proposes further points at which estradiol wields an influence in neuroprotection against cerebral ischemia.

Postischemic inflammation strongly contributes to the extent of cerebral injury, and $17 \beta$-estradiol may exert protection through anti-inflammatory (Figure 1) actions (Vegeto et al., 2008; Suzuki et al., 2009). In fact, the presence of initial neural inflammation is negatively correlated with serum estradiol levels (Wang et al., 2007). The proposed antiinflammatory action of estradiol is strengthened by findings that $17 \beta$-estradiol is neuroprotective when administered immediately upon ovariectomy but not when administered after 10 weeks of hypoestrogenicity, demonstrating that a prolonged period of hypoestrogenicity disrupts not only the neuroprotective but also the anti-inflammatory actions of estradiol (Suzuki et al., 2007, 2009). The first cellular response in inflammation is the activation and accumulation of neutrophils (Morganti-Kossmann et al., 2001). It is evident in models of transient cerebral ischemia that tissue loss is reduced significantly through the inhibition of neutrophil infiltration, as it is apparent that neutrophils wield the most damage to ischemic lesions once reperfusion is undertaken (Connolly et al., 1996; Guha and Mackman, 2001; Wang et al., 2007). It would seem that neutrophil accumulation is also negatively correlated with serum estradiol levels (Liao et al., 2001), strengthening evidence for the neuroprotective role of the female sex hormone even more. The anti-inflammatory properties of $17 \beta$-estradiol in the cerebral circulation thus influence the incidence, outcome, and severity of injury in stroke

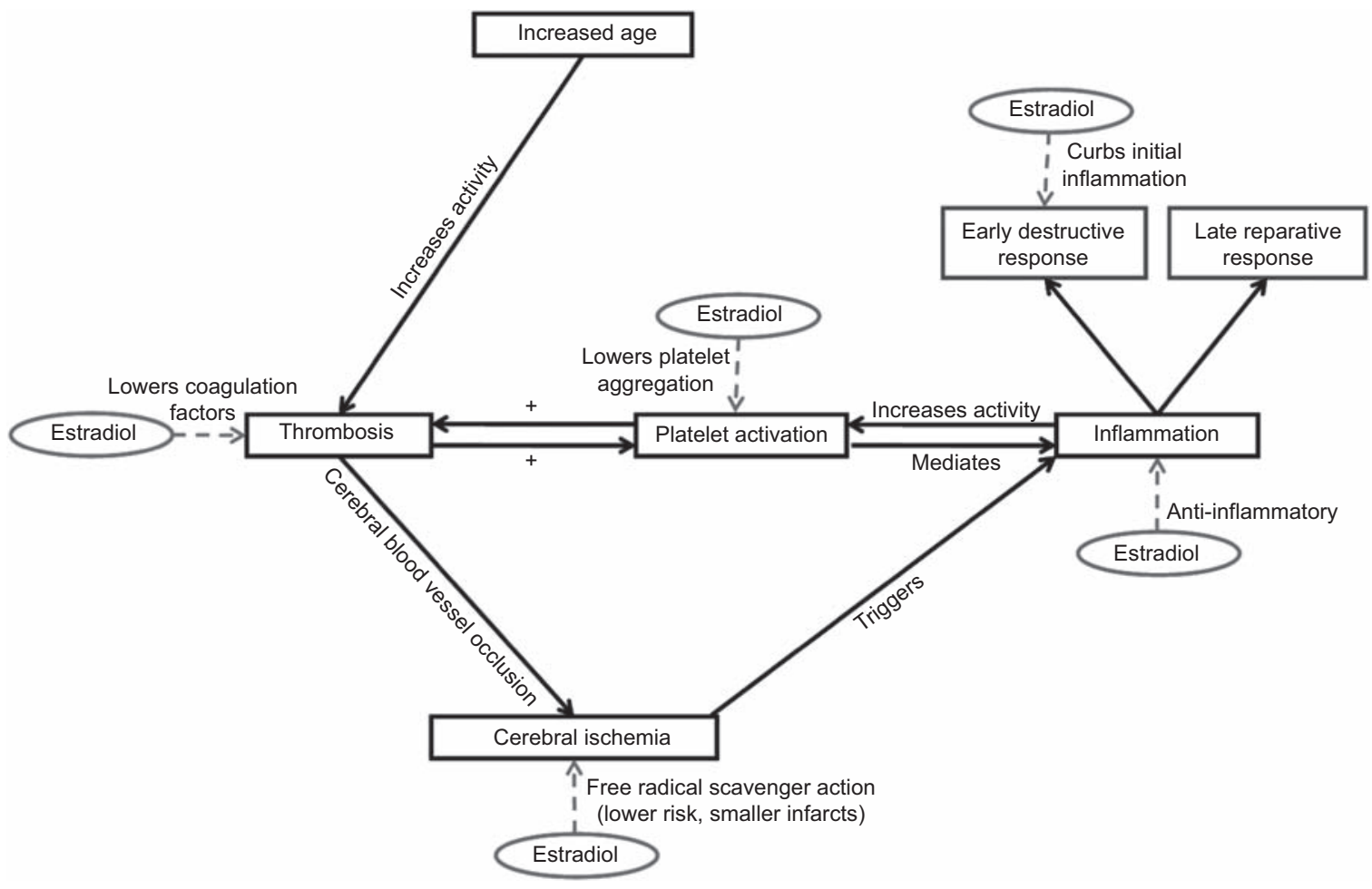

Figure 1 Possible points of estradiol's influence in neuroprotection against cerebral ischemia. Estradiol affects thrombosis activity through the lowering of coagulation factors and platelet aggregation. It displays anti-inflammatory mechanisms and lowers the negative consequences of cerebral infarction through free radical scavenger action. 
by attenuating ischemia-induced inflammatory responses (Suzuki et al., 2009).

Not only does estradiol exert protective anti-inflammatory actions subsequent to cerebral ischemia, but it also has a role in thrombosis (Figure 1) regulation (Wong et al., 2008; Bailey et al., 2009), the alteration of which may result in cerebral ischemia. Coagulation factors and proteins are lowered by the presence of estrogen, though some factors (i.e., fibrinogen) are inherently higher in females than in males (Mendelsohn and Karas, 1999; Bailey et al., 2009); in addition, there are cyclic patterns in these coagulation proteins that correspond to the menstrual cycle in females (Kadir et al., 1999; Bailey et al., 2009). Functionally, female platelet isolates, though capable of binding more fibrinogen and displaying a greater maximal aggregation extent than male platelet isolates (Leng et al., 2004; Bailey et al., 2009), actually do not aggregate as quickly as the larger number of male platelets do, thus to some degree rendering females less susceptible to thrombosis. In the absence of estradiol in acyclicity, female platelets are again more susceptible to thrombosis (Wong et al., 2008; Bailey et al., 2009). Platelets are indeed found to express $\mathrm{ER} \beta$, which is hypothesized to have a direct effect on platelet function (Peters et al., 2002; Jayachandran and Miller, 2003; Bailey et al., 2009). Thus, it becomes clear that there are not only sex-based differences in coagulation processes but also age-based differences; as in a hypoestrogenic state, females not only have higher coagulation factors but also higher maximal platelet aggregation capabilities than males, rendering them more prone to thrombosis in an acyclic state.

Finally, it must be noted that estradiol replacement is not universally neuroprotective. It has been suggested that the neuroprotective effects of estrogen are more evident in transient than in permanent models of cerebral ischemia (Macrae and Carswell, 2006; Selvamani and Sohrabji, 2010). This is suggested due to findings that in severe ischemic injury, there are no sex differences in infarct size and also no reduction of the infarct with 17 $\beta$-estradiol administration (Vergouwen et al., 2000; Selvamani and Sohrabji, 2010). Permanent ischemia leads to severe metabolic impairment in the cerebral cortex, which results in necrosis of many neurons in the region within several hours following injury. Regions surrounding the core of ischemia can be salvaged from apoptosis through the powerful neuroprotective action of $17 \beta$-estradiol (Prewitt and Wilson, 2007; Suzuki et al., 2009), but the effects of estradiol at the ischemic core are only visible in transient ischemic models. Conclusively, 17ß-estradiol protects the brain through suppression of neuronal apoptosis during the initial 24 hours after injury, in part by suppressing the inflammatory response, and enhances neurogenesis within the first 96 hours after ischemic stroke (Suzuki et al., 2009).

\section{Conclusions}

Platelets are central to both thrombosis and inflammation. Not only is platelet localization essential to thrombus initiation, formation, and stabilization, but in areas of ischemia/reperfusion injury in cerebral ischemia, platelets and leukocytes colocalize, linking hemostatic thrombotic and inflammatory responses. Estrogen is neuroprotective in a myriad of mechanisms - a few of which include anti-inflammatory actions and regulation of coagulation factors affecting thrombosis ability. The cascade of cerebral ischemia, its association to inflammation and thrombosis, and their connection to each other are thus intricately intertwined.

\section{References}

Alkayed, N.J., Harukuni, I., Kimes, A.S., London, E.D., Traystman, R.J., and Hurn, P.D. (1998). Gender-linked brain injury in experimental stroke. Stroke 29, 159-165.

Alkayed, N.J., Murphy, S.J., Traystman, R.J., and Hurn, P.D. (2000). Neuroprotective effects of female gonadal steroids in reproductively senescent female rats. Stroke 31, 161-168.

Altman, R. (2003). Risk factors in coronary atherosclerosis atheroinflammation: the meeting point. Thromb. J. 1, 1-11.

André, P., Prasad, K.S., Denis, C.V., He, M., Papalia, J.M., Hynes, R.O., Phillips, D.R., and Wagner, D.D. (2002). CD40L stabilizes arterial thrombi by a $\beta 3$ integrin - dependent mechanism. Nat. Med. 8, 247-252.

Bailey, A.L., Scantlebury, D.C., and Smyth, S.S. (2009). Thrombosis and antithrombotic therapy in women. Arterioscler. Thromb. Vasc. Biol. 29, 284-288.

Bake, S. and Sohrabji, F. (2004). 17ß-Estradiol differentially regulates blood-brain barrier permeability in young and aging female rats. Endocrinology 145, 5471-5475.

Barone, F.C., Hillegass, L.M., Tzimas, M.N., Schmidt, D.B., Foley, J.J., White, R.F., Price, W.J., Feuerstein, G.Z., Clark, R.K., Griswold, D.E., et al. (1995). Time-related changes in myeloperoxidase activity and leukotriene B4 receptor binding reflect leukocyte influx in cerebral focal stroke. Mol. Chem. Neuropathol. 24, 13-30.

Bar-Shavit, R., Kahn, A.J., Mann, K.G., and Wilner, G.D. (1986). Identification of a thrombin sequence with growth factor activity on macrophages. Proc. Natl. Acad. Sci. USA 83, 976-980.

Braeuninger, S. and Kleinschnitz, C. (2009). Rodent models of focal cerebral ischemia: procedural pitfalls and translational problems. Exp. Transl. Stroke Med. 25, 1-8.

Brand, K., Fowler, B.J., Edgington, T., and Mackman, N. (1991). Tissue factor mRNA in THP-1 monocytic cells is regulated at both transcriptional and posttranscriptional levels in response to lipopolysaccharide. Mol. Cell Biol. 11, 4732-4738.

Brouns, R. and De Deyn, P.P. (2009). The complexity of neurobiological processes in acute ischemic stroke. Clin. Neurol. Neurosurg. 111, 483-495.

Burstein, S.A. (1997). Cytokines, platelet production and hemostasis. Platelets 8, 93-104.

Butcher, E.C. (1991). Leukocyte-endothelial cell recognition: three (or more) steps to specificity and diversity. Cell 67, 1033-1036.

Butenas, S. and Mann, K.G. (2002). Blood coagulation. Biochemistry 67, 3-12.

Camera, M., Giesen, P.L., Fallon, J., Aufiero, B.M., Taubman, M., Tremoli, E., and Nemerson, Y. (1999). Cooperation between VEGF and TNF- $\alpha$ is necessary for exposure of active tissue factor on the surface of human endothelial cells. Arterioscler. Thromb. Vasc. Biol. 19, 531-537.

Carswell, H.V., Dominiczak, A.F., and Macrae, I.M. (2000). Estrogen status affects sensitivity to focal cerebral ischemia in stroke- 
prone spontaneously hypertensive rats. Am. J. Physiol. Heart Circ. Physiol. 278, H290-H294.

Connolly Jr., E.S., Winfree, C.J., Springer, T.A., Naka, Y., Liao, H., Yan, S.D., Stern, D.M., Solomon, R.A., Gutierrez-Ramos, J.C., and Pinsky, D.J. (1996). Cerebral protection in homozygous null ICAM-1 mice after middle cerebral artery occlusion. Role of neutrophil adhesion in the pathogenesis of stroke. J. Clin. Invest. 97, 209-216.

Danesh, J., Lewington, S., Thompson, S.G., Lowe, G.D.O., Collins, R., Kostis, J.B., Wilson, A.C., Folsom, A.R., Wu, K., Benderly, M., et al. (2005). Plasma fibrinogen level and the risk of major cardiovascular diseases and nonvascular mortality: an individual participant meta-analysis. J. Am. Med. Assoc. 294, 1799-1809.

Danton, G.H. and Dietrich, W.D. (2003). Inflammatory mechanisms after ischemia and stroke. J. Neuropathol. Exp. Neurol. 62, 127-136.

Davis, M., Mendelow, A.D., Perrry, R.H., Chambers, I.R., and James, O.F. (1995). Experimental stroke and neuroprotection in the aging rat brain. Stroke 26, 1072-1078.

Day, S.M., Reeve, J.L., Pedersen, B., Farris, D.M., Myers, D.D., Im, M., Wakefield, T.W., Mackman, N., and Fay, W.P. (2005). Macrovascular thrombosis is driven by tissue factor derived primarily from the blood vessel wall. Blood 105, 192-198.

Demopoulos, H.B., Milvy, P., Kakari, S., and Ransohoff, J. (1972). Molecular aspects of membrane structure in cerebral edema. In: Steroids and Brain Edema, H. Reulen and K. Shurmann, eds. (Springer-Verlag, New York), pp. 29-39.

Diacovo, T.G., Roth, S.J., Buccola, J.M., Bainton, D.F., and Springer, T.A. (1996). Neutrophil rolling, arrest, and transmigration across activated, surface-adherent platelets via sequential action of P-selectin and the $\beta 2$-integrin CD11b/CD18. Blood 88 , 146-157.

Drača, S. (2009). Are female sex steroids neuroprotective in experimental stroke. Med. Hypotheses 73, 1051-1052.

Drake, T.A., Morrissey, J.H., and Edgington, T.S. (1989). Selective cellular expression of tissue factor in human tissues: implications for disorders of hemostasis and thrombosis. Am. J. Pathol. 134, 1087-1097.

Dubal, D.B., Kashon, M.L., Pettigrew, L.C., Ren, J.M., Finkelstein, S.P., Rau, S.W., and Wise, P.M. (1998). Estradiol protects against ischemic injury. J. Cereb. Blood Flow Metab. 18, 1253-1258.

Dubal, D.B., Zhu, H., Yu, J., Rau, S.W., Shughrue, P.J., Merchenthaler, I., Kindy, M.S., and Wild, P.M. (2001). Estrogen receptor $\alpha$, not $\beta$ is a critical link in estradiol-mediated protection against brain injury. Proc. Natl. Acad. Sci. USA 98, 1952-1957.

Elkins, J.S. and Johnston, S.C. (2003). Thirty-year projections for details from ischemic stroke in the United States. Stroke 34, 2109-2112.

Emms, H. and Lewis, G.P. (1985). Sex and hormonal influences on platelet sensitivity and coagulation in the rat. Br. J. Pharmacol. 86, 557-563.

Esmon, C.T. (2003). Inflammation and thrombosis. J. Thromb. Haemost. 1, 1343-1348.

Fatah, K., Hamsten, A., Blombäck, B., and Blombäck, M. (1992). Fibrin gel network characteristics and coronary heart disease: relations to plasma fibrinogen concentration, acute phase protein, serum lipoproteins and coronary atherosclerosis. Thromb. Haemost. 68, 130-135.

Frenette, P.S., Denis, C.V., Weiss, L., Jurk, K., Subbarao, S., Kehrel, B., Hartwig, J.H., Vestweber, D., and Wagner, D.D. (2000). P-Selectin glycoprotein ligand 1 (PSGL-1) is expressed on platelets and can mediate platelet-endothelial interactions in vivo. J. Exp. Med. 191, 1413-1422.
Furman, M.I., Benoit, S.E., Barnard, M.R., Valeri, C.R., Borbone, M.L., Becker, R.C., Hechtman, H.B., and Michelson, A.D. (1998). Increased platelet reactivity and circulating monocyte-platelet aggregates in patients with stable coronary artery disease. J. Am. Coll. Cardiol. 31, 352-358.

Gibson, C.L., Constantin, D., Prior, M.J., Bath, P.M., and Murphy, S.P. (2005). Progesterone suppresses the inflammatory response and nitric oxide synthase-2 expression following cerebral ischemia. Exp. Neurol. 193, 522-530.

Gimbrone Jr., M.A. (1995). Vascular endothelium: an integrator of pathophysiologic stimuli in atherosclerosis. Am. J. Cardiol. 75, B67-B70.

Goel, M.S. and Diamond, S.L. (2001). Neutrophil enhancement of fibrin deposition under flow through platelet-dependent and -independent mechanisms. Arterioscler. Thromb. Vasc. Biol. 21, 2093-2098.

Guha, M. and Mackman, N. (2001). LPS induction of gene expression in human monocytes. Cell Signal. 13, 85-94.

Hallenbeck, J.M. (1996). Significance of the inflammatory response in brain ischemia. Acta Neurochir. 66, S27-S31.

Hawiger, J. (1995). Mechanisms involved in platelet vessel wall interaction. Thromb. Haemost. 74, 369-372.

Hawk, T., Zhang, Y.Q., Rajakumar, G., Day, A.L., and Simpkins, J.W. (1998). Testosterone increases and estradiol decreases middle cerebral artery occlusion lesion size in male rats. Brain Res. 796, 296-298.

Henn, V., Slupsky, J.R., Gräfe, M., Anagnostopoulos, I., Förster, R., Müller-Berghaus, G., and Kroczek, R.A. (1998). CD40 ligand on activated platelets triggers an inflammatory reaction of endothelial cells. Nature 391, 591-594.

Herd, C.M. and Page, C.P. (1994). Pulmonary immune cells in health and disease: platelets. Eur. Respir. J. 7, 1145-1160.

Hynes, R.O. (1990). Fibronectins (Springer-Verlag, New York).

Jayachandran, M. and Miller, V.M. (2003). Human platelets contain estrogen receptor $\alpha$, caveolin-1 and estrogen receptor associated proteins. Platelets $14,75-81$.

Jezierski, M.K. and Sohrabji, F. (2001). Neurotrophin expression in the reproductively senescent forebrain is refractory to estrogen stimulation. Neurobiol. Aging 22, 309-319.

Jia, J., Guan, D., Zhu, W., Alkayed, N.J., Wang, M.M., Hua, Z., and $\mathrm{Xu}, \mathrm{Y}$. (2009). Estrogen inhibits Fas-mediated apoptosis in experimental stroke. Exp. Neurol. 215, 48-52.

Kadir, R.A., Economides, D.L., Sabin, C.A., Owens, D., and Lee, C.A. (1999). Variations in coagulation factors in women: effects of age, ethnicity, menstrual cycle and combined oral contraceptive. Thromb. Haemost. 82, 1456-1461.

Konstantopoulos, K., Neelamegham, S., Burns, A.R., Hentzen, E., Kansas, G.S., Snapp, K.R., Berg, E.L., Hellums, J.D., Smith, C.W., McIntire, L.V., et al. (1998). Venous levels of shear support neutrophil-platelet adhesion and neutrophil aggregation in blood via P-selectin and $\beta 2$-integrin. Circulation 98, 873-882.

Kranzhöfer, R., Clinton, S.K., Ishii, K., Coughlin, S.R., Fenton 2nd, J.W., and Libby, P. (1996). Thrombin potently induces cytokine production by human vascular smooth muscle cells but not in mononuclear phagocytes. Circ. Res. 79, 286-294.

Kuijper, P.H., Gallardo Torres, H.I., van der Linden, J.A., Lammers, J.W., Sixma, J.J., Koenderman, L., and Zwaginga, J.J. (1996). Platelet-dependent primary hemostasis promotes selectin- and integrin-mediated neutrophil adhesion to damaged endothelium under flow conditions. Blood 87, 3271-3281.

Laursen, J.B., Somers, M., Kurz, S., Warnholtz, A., McCann, L., Freeman, B.A., Tarpley, M., Fukai, T., and Harrison, D.G. (2001). Endothelial regulation of vasomotion in apoE-deficient 
mice: implications for interactions between peroxynitrite and tetrahydrobiopterin. Circulation 103, 1282-1288.

Leng, X.H., Hong, S.Y., Larrucea, S., Zhang, W., Li, T.T., López, J.A., and Bray, P.F. (2004). Platelets of female mice are intrinsically more sensitive to agonists than are platelets of males. Arterioscler. Thromb. Vasc. Biol. 24, 376-381.

Liao, S.L., Chen, W.Y., Kuo, J.S., and Chen, C.J. (2001). Association of serum estrogen level and ischemic neuroprotection in female rats. Neurosci. Lett. 297, 159-162.

Libby, P. and Simon, D.I. (2001). Inflammation and thrombosis: the clot thickens. Circulation 130, 1718-1720.

Lorant, D.E., Patel, K.D., McIntyre, T.M., McEver, R.P., Prescott, S.M., and Zimmerman, G.A. (1991). Coexpression of GMP-140 and PAF by endothelium stimulated by histamine or thrombin: a juxtacrine system for adhesion and activation of neutrophils. J. Cell Biol. 115, 223-224.

Lowe, G.D.O., Rumley, A., Woodward, M., Morrison, C.E., Philippou, H., Lane, D.A., and Tunstall-Pedoe, H. (1997). Epidemiology of coagulation factors, inhibitors and activation markers: the Third Glasgow MONICA Survey. I. Illustrative reference ranges by age, sex and hormone use. Br. J. Haematol. 97, 775-784.

Lowe, G.D.O., Rumley, A., and Mackie, I.J. (2004). Plasma fibrinogen. Ann. Clin. Biochem. 41, 430-440.

Mackman, N. (2004). Role of tissue factor in hemostasis, thrombosis, and vascular development. Arterioscler. Thromb. Vasc. Biol. 24, 1015-1022.

Macrae, I.M. and Carswell, H.V. (2006). Oestrogen and stroke: the potential for harm as well as benefit. Biochem. Soc. Trans. 34, 1362-1365.

Mann, K.G., van't Veer, C., Cawthern, K., and Butenas, S. (1998). The role of tissue factor pathway in initiation of coagulation. Blood Coagul. Fibrinolysis 9, S3-S7.

Manthey, D. and Behl, C. (2006). From structured biochemistry to expression profiling: neuroprotective activities of estrogen. Neuroscience 138, 845-850.

McCullough, L.D. and Hurn, P.D. (2003). Estrogen and ischemic neuroprotection: an integrated view. Trends Endocrinol. Metab. 14, 228-235.

Mendelsohn, M.E. and Karas, R.H. (1999). The protective effects of estrogen on the cardiovascular system. N. Engl. J. Med. 340, 1801-1811.

Miller, D.L., Yaron, R., and Yellin, M.J. (1998). CD40L-CD40 interactions regulate endothelial cell surface tissue factor and thrombomodulin expression. J. Leukoc. Biol. 63, 373-379.

Mitka, M. (2006). Studies explore stroke's gender gap. J. Am. Med. Assoc. 295, 1755-1756.

Morganti-Kossmann, M.C., Rancan, M., Otto, V.I., Stahel, P.F., and Kossmann, T. (2001). Role of cerebral inflammation after traumatic brain injury: a revisited concept. Shock 16, 165-177.

Myers, D.D. and Wakefield, T.W. (2005). Inflammation-dependent thrombosis. Front. Biosci. 10, 2750-2757.

Nathan, C. (2002). Points of control in inflammation. Nature 420, $846-852$.

Nemerson, Y. (1988). Tissue factor and hemostasis. Blood 71, 1-8.

Ni, H., Denis, C.V., Subbarao, S., Degen, J.L., Sato, T.N., Hynes, R.O., and Wagner, D.D. (2000). Persistence of platelet thrombus formation in arterioles of mice lacking both von Willebrand factor and fibrinogen. J. Clin. Invest. 106, 385-392.

Ostrovsky, L., King, A.J., Bond, S., Mitchell, D., Lorant, D.E., Zimmerman, G.A., Larsen, R., Niu, X.F., and Kubes, P. (1998). A juxtacrine mechanism for neutrophil adhesion on platelets involves platelet-activating factor and a selectin-dependent activation process. Blood 91, 3028-3036.
Ott, I., Neumann, F.J., Gawaz, M., Schmitt, M., and Schömig, A. (1996). Increased neutrophil-platelet adhesion in patients with unstable angina. Circulation 94, 1239-1246.

Park, E.M., Cho, S., Frys, K.A., Glickstein, S.B., Zhou, P., Anrather, J., Ross, M.E., and Iadecola, C. (2006). Inducible nitric oxide synthase contributes to gender differences in ischemic brain injury. J. Cereb. Blood Flow Metab. 26, 392-401.

Pendurthi, U.R., Alok, D., and Rao, L.V.M. (1997). Binding of factor VIIa to tissue factor induces alterations in gene expression in human fibroblast cells: up-regulation of poly(A) polymerase. Proc. Natl. Acad. Sci. USA 94, 12598-12603.

Peters, L.L., Cheever, E.M., Ellis, H.R., Magnani, P.A., Svenson, K.L., Von Smith, R., and Bogue, M.A. (2002). Large-scale, highthroughput screening for coagulation and hematologic phenotypes in mice. Physiol. Genomics 11, 185-193.

Pretorius, E., Briedenhann, S., Marx, J., and Franz, R.C. (2006). Structural changes in the fibrin network of a pretoria family with dysfibrinogenemia: a scanning electron microscopical study. Ultrastruct. Pathol. 30, 167-176.

Pretorius, E., Briedenhann, S., Marx, J., Smit, E., Van Der Merwe, C.F., Pieters, M., and Franz, R.C. (2007). Ultrastructural comparison of the morphology of three different platelet and fibrin fibre preparations. Anat. Rec. (Hoboken). 290, 188-198.

Prewitt, A.K. and Wilson, M.E. (2007). Changes in estrogen receptor- $\alpha$ mRNA in the mouse cortex during development. Brain Res. 1134, 62-69.

Rauch, U. and Nemerson, Y. (2000). Tissue factor, the blood, and the arterial wall. Trends Cardiovasc. Med. 10, 139-143.

Ross, R. (1985). Platelets, platelet-derived growth factor, growth control, and their interactions with the vascular wall. J. Cardiovasc. Pharmacol. 7, S186-S190.

Roth, G.J. (1992). Platelets and blood vessels: the adhesion event. Immunol. Today 13, 100-105.

Saenger, A.K. and Christenson, R.H. (2010). Stroke biomarkers: progress and challenges for diagnosis, prognosis, differentiation, and treatment. Clin. Chem. 56, 21-33.

Sakai, T., Johnson, K.J., Murozono, M., Sakai, K., Magnuson, M.A., Wieloch, T., Cronberg, T., Isshiki, A., Erickson, H.P., and Fässler, R. (2001). Plasma fibronectin supports neuronal survival and reduces brain injury following transient focal cerebral ischemia but is not essential for skin-wound healing hemostasis. Nat. Med. 3, 324-330.

Schecter, A.D., Giesen, P.L., Taby, O., Rosenfield, C.L., Rossikhina, M., Fyfe, B.S., Kohtz, D.S., Fallon, J.T., Nemerson, Y., and Taubman, M.B. (1997). Tissue factor expression in human arterial smooth muscle cells. TF is present in three cellular pools after growth factor stimulation. J. Clin. Invest. 100, 2276-2285.

Selvamani, A. and Sohrabji, F. (2010). Reproductive age modulates the impact of focal ischemia on the forebrain as well as the effects of estrogen treatment in female rats. Neurobiol. Aging 31, $1618-1628$.

Shebuski, R.J. and Kilgore, K.S. (2002). Role of inflammatory mediators in thrombogenesis. J. Pharmacol. Exp. Ther. 300, 729-735.

Simpkins, J.W., Rajakumar, G., Zhang, Y.Q., Simpkins, C.E., Greenwald, D., Yu, C.J., Bodor, N., and Day, A.L. (1997). Estrogens may reduce mortality and ischemic damage caused by middle cerebral artery occlusion in the female rat. J. Neurosurg. 87, 724-730.

Singer, C.A., Rogers, K.L., Strickland, T.M., and Dorsa, D.M. (1996). Estrogen protects primarily cortical neurons from glutamate toxicity. Neurosci. Lett. 212, 13-16. 
Springer, T.A. (1994). Traffic signals for lymphocyte recirculation and leukocyte emigration: the multistep paradigm. Cell 76, 301-314.

Stewart, G.J., Ritchie, W.G.M., and Lynch, P.R. (1974). Venous endothelial damage produced by massive sticking and emigration of leukocytes. Am. J. Pathol. 74, 507-532.

Suzuki, S., Brown, C.M., Dela Cruz, C.D., Yang, E., Bridwell, D.A., and Wise, P.M. (2007). Timing of estrogen therapy after ovariectomy dictates the efficacy of its neuroprotective and antiinflammatory actions. Proc. Natl. Acad. Sci. USA 104, 6013-6018.

Suzuki, S., Brown, C.M., and Wise, P.M. (2009). Neuroprotective effects of estrogens following ischemic stroke. Front Neuroendocrinol. 30, 201-211.

Taylor Jr., F.B., Chang, A., Esmon, C.T., D’Angelo, A., Vigano-D' Angelo, S., and Blick, K.E. (1987). Protein C prevents the coagulopathic and lethal effects of Escherichia coli infusion in the baboon. J. Clin. Invest. 79, 918-925.

ten Cate, J.W., van der Poll, T., Levi, M., ten Cate, H., and van Deventer, S.J. (1997). Cytokines: triggers of clinical thrombotic disease. J. Thromb. Haemost. 87, 415-419.

Toung, T.J.K., Traystman, R.J., and Hurn, P.D. (1998). Estrogenmediated neuroprotection after experimental stroke in male rats. Stroke 29, 1666-1670.

Varma, M.R., Varga, A.J., Knipp, B.S., Sukheepod, P., Upchurch, G.R., Kunkel, S.L., Wakefield, T.W., and Henke, P.K. (2003). Neutropenia impairs venous thrombosis resolution in the rat. J. Vasc. Surg. 38, 1090-1098.

Vegeto, E., Benedusi, V., and Maggi, A. (2008). Estrogen anti-inflammatory activity in brain: a therapeutic opportunity for menopause and neurodegenerative diseases. Front. Neuroendocrinol. 29, 507-519.

Vergouwen, M.D., Anderson, R.E., and Meyer, F.B. (2000). Gender differences and the effects of synthetic exogenous and non-synthetic estrogens in focal cerebral ischemia. Brain Res. $\underline{878,88-97 .}$

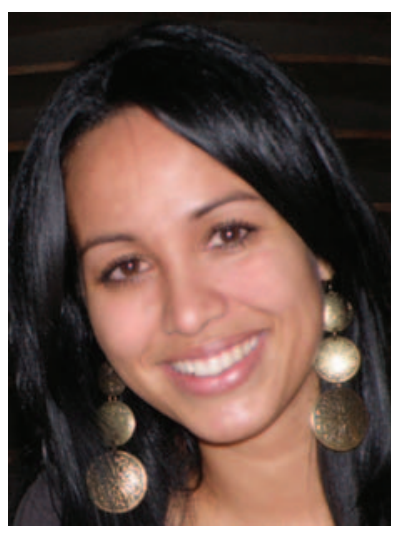

Wendy Jeannette van der Spuy is PhD student with the Department of Anatomy's Division of Histology and Cell Biology at the University of Pretoria, South Africa. She has a passion for electron microscopy, biological research and scientific writing. She is credited as primary or co-author of 14 scientific publications since 2009, with a further number of articles in the peer-review process for 2012. Together with Professor Etheresia Pretorius, her supervisor, she wishes to continue in the field of stroke and inflammatory research.
Wagner, D.D. and Burger, P.C. (2003). Platelets in inflammation and thrombosis. Arterioscler. Thromb. Vasc. Biol. 23, 2131-2137.

Wakefield, T.W., Strieter, R.M., Prince, M.R., Downing, L.J., and Greenfield, L.J. (1997). Pathogenesis of venous thrombosis: a new insight. Cardiovasc. Surg. 5, 6-15.

Wang, Q., Tang, X.N., and Yenari, M.A. (2007). The inflammatory response in stroke. J. Neuroimmunol. 184, 53-68.

Weber, C. and Springer, T.A. (1997). Neutrophil accumulation on activated, surface-adherent platelets in flow is mediated by interaction of Mac-1 with fibrinogen bound to $\alpha \operatorname{IIb} \beta 3$ and stimulated by platelet-activating factor. J. Clin. Invest. 100, 2085-2093.

White, J.G. (1987). Views of the platelet cytoskeleton at rest and at work. Ann. NY Acad. Sci. 509, 156-176.

Wilcox, J.N., Smith, K.M., Schwartz, S., and Gordon, D. (1989). Localization of tissue factor in the normal vessel wall and in the atherosclerotic plaque. Proc. Natl. Acad. Sci. USA 86, $2839-2843$.

Wohner, N. (2008). Role of cellular elements in thrombus formation and dissolution. Cardiovasc. Hematol. Agents Med. Chem. 6, 224-228.

Wolf, P.A. (1990). An overview of the epidemiology of stroke. Stroke 21, S114-S116.

Wong, J.H., Dukes, J., Levy, R.E., Sos, B., Mason, S.E., Fong, T.S., and Weiss, E.J. (2008). Sex differences in thrombosis in mice are mediated by sex-specific growth hormone secretion patterns. J. Clin. Invest. 118, 2969-2978.

Zhang, X., Selli, M.L., Baglioni, S., Hauri, A., Chiari, R., Dottorini, M., Todisco, T., Nenci, G.G., and Gresele, P. (1993). Platelets from asthmatic patients migrate in vitro in response to allergen stimulation. Thromb. Haemost. 69, 1356.

Received February 16, 2012; accepted March 5, 2012

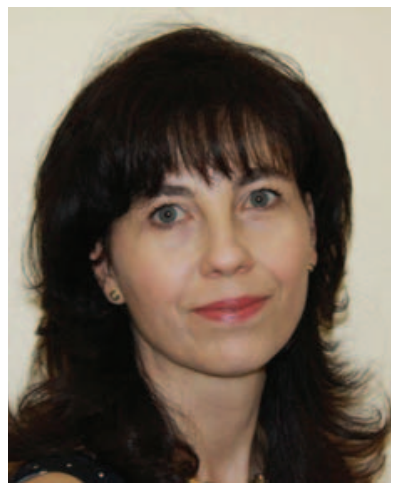

Etheresia (Resia) Pretorius received her $\mathrm{PhD}$ in 1998, and is a Professor in the Department of Physiology, University of Pretoria, South Africa. She has published over 150 peer reviewed manuscripts and book chapters and, since 2000, has also been mentor to 28 successful MSc and $\mathrm{PhD}$ students. Her focus is diseases associated with inflammation and she combines ultrastructure and electron microscopy with physiology. Particularly, her focus is the effect of inflammation on platelets, red blood cells and the coagulation system. In December 2011, Resia was named the Southern African Regional winner in the category, Basic Science, Technology and Innovation, of the prestigious African Union Kwame Nkrumah Scientific Awards, which forms part of the African Union Scientific Awards Programme. 